\title{
Performance Improvement of Outage Users at Cell Edges Through Cognitive-Femtocell Deployment Over Macrocell Network
}

\author{
Joydev Ghosh \\ Dept. of Electronics and Tele-Communication Engg. \\ The New Horizons Institute of Technology \\ Durgapur, West Bengal (W.B), INDIA \\ Joydev.ghosh.ece@gmail.com
}

\author{
Sanjay Dhar Roy \\ Dept. of Electronics and Communication Engg. \\ National Institute of Technology \\ Durgapur, West Bengal (W.B), INDIA \\ s_dharroy@yahoo.com
}

\begin{abstract}
This research focuses on the problem of cell edge user coverage in the context of femtocell networks operating within the locality of macrocell border. The macro cell-edge users get assisted by the cognitive-femto base station (FBS) to receive a consistent quality of service $(\mathrm{QoS})$ because of their long distance from the macro base station (MBS). Considering various environment factors such as wall structure, number of walls, distance between macrocell and users, interference effect (i.e., cotier and cross-tier), we compute not only the overall outage probability of single input single output (SISO) system and single input multiple output (SIMO) system users but also based on subcarrier and power allocation the performance of the two tier network is analysed with macrocell throughput as the performance metric. Finally, the effectiveness of the scheme is verified by extensive matlab simulation.
\end{abstract}

Keywords-Cognitive Femtocell Networks, Okumura-Hata Propagation Pathloss Model (Empirical Model), SISO and SIMO users, Outage Probability, Macrocell Throughput.

\section{I.INTRODUCTION}

Several recent studies show that more than $70 \%$ of data traffic is generated indoors [1]. However, mobile users in indoor environments may have difficulty in receiving high speed services facility from outdoor macro base station (MBS) due to the penetration loss. The motion of a user from cell centre to cell edge results in power reduction of the signal transmitted from the cell centre which increases the interference effect. This dead zone problem can be mitigated by encompassing small base stations which are known as femtocell network [2], which is considered in the next generation wireless communication systems such as 3GPP (3rd Generation Partnership Project) Long-Term Evolution(LTE) and mobile WiMAX (IEEE 802.16m) [4]. The LTE (Long Term Evolution) has been developed by 3GPP (3rd Generation Partnership Project) to bridge the transition from $3 \mathrm{G}$ to $4 \mathrm{G}$. Currently, $4 \mathrm{G}$ has been launched to address modern loopholes of wireless communications. The next phase of development will be commencing soon which is unofficially named as $5 \mathrm{G}$ to take the remodelling steps ahead where LTE re-engineers the OFDMA to generate a high-speed radio interface [6]. An LTE network segregates the spectrum into Resource Blocks (RBs). RBs are the smallest allocated resource unit [8] which is also time frequency unit. If recurrent reports from a User Equipment (UE) make sure of interference effect with the allocated $\mathrm{RB}$, then some other $\mathrm{RB}$ can be randomly re-assigned to the UE. This provision is successful in low-traffic and less dense situations. For massive traffic, a co-ordinated scheme is preferred; otherwise the reallocation may only result in a randomized block already being used by others. Here, we address quite a few shortcomings still holding in the current stage of networking and emphasis on some design considerations of $5 \mathrm{G}$ embracing femtocells and cognitive radio technology. "Femto Cell" is a low power radio access point which operates in both licensed and unlicensed spectrum and it offers network coverage from 10 meters to several hundred meters. Femto access point (FAP) has equivalent functions as like macro base station (MBS) e.g., communicating with the user equipments (UEs) and setting up a voice circuit with the cognitive radio network (CRN) [1]. The user equipment which takes the help of macro base station (MBS) network for communication is called macro user equipment (MUE) or macro base station user (MBSU). Similarly, the equipment which uses the network of femto base station (FBS) is called femto user equipment (FUE) or femto base station user (FBSU). The deployment of dual-tier (heterogeneous) networks aiding macro and femtocells co-existance on the same spectrum (licensed or unlicensed) and in the same geographical area invokes new technical challenges to realize the idea. The new technical challenges are inter-tier and intra-tier interference; coverage holes due to huge transmit power discrepancy across femto cells, handoff optimization and heterogeneous backhaul design [3].

As femtocells are self-organized, Femto Access Point (FAP) needs to have the capability to identify the transmission environment and adjust their configurations intelligently. Cognitive radio (CR) is an effective technique to provide the intelligence to FAPs. CR enhanced femtocells can obtain the information of macrocells by spectrum sensing and access the channels without interrupting macrocell users, especially the "isolated" users without femtocell access. In other words, the link between macro base station (MBS) and macro user equipment (MUE) is treated as the primary link with high priority, while the link between femto access point (FAP) and femto user equipment (FUE) is the secondary link utilizing the spectrum in an opportunistic way. The intelligence provided by CR makes it possible for femtocells to avoid or restrict the mutual interference with macrocells in a distributed manner [8].

In this paper, we will have to focus on femto access point (FAP) because of two major reasons. Firstly, the co-tier interference is more eminent to FAPs due to the large transmit 
power difference between the FAPs and MBSs. Secondly, we arrest the impact of outage at the cell edges on overall performance of the networks which can be resolved by implantation of the FAPs, such as the closed access system and the open access system.

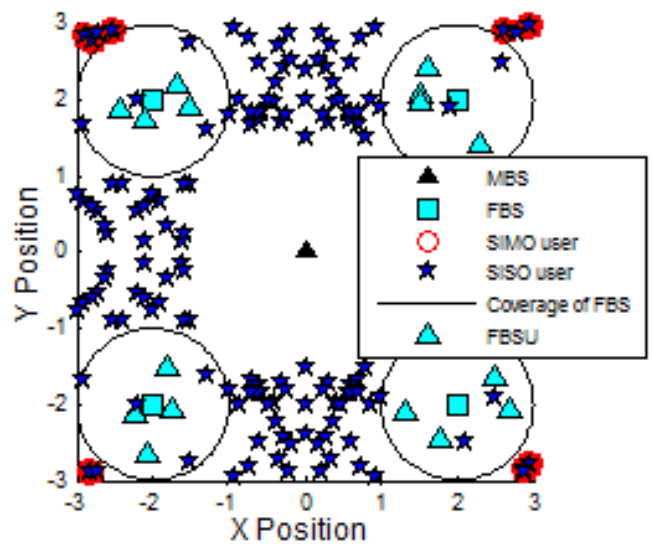

Fig.1 Co-existance scenario of FAP and Macrocell in our proposed network.

Ultimately, as FAPs are principally deployed by the subscribers, cognition is expected to be an inherent property that should be executed in all FAPs. In practical downlink transmission most of the handsets can only accommodate one or at most two antennas. Here, Single input single output (SISO) system and single input multiple output (SIMO) systems come into consideration. In single input single output (SISO) system, the transmitter has a single antenna and the receiver also has single antenna. There is no diversity and no additional processing required. The single input multiple output (SIMO) system where the transmitter has a single antenna and the receiver has multiple antennas. It is often used to enable a receiver system that receives signals from a number of independent sources to combat the effects of fading. The major contributions of this paper are highlighted below-

- A simplified network model for resource allocation in CR based femtocell network has been introduced.

- A simulation model has been proposed to evaluate the performance of cell edge users. A cell edge user is an underprivileged user which is not having good QoS normally.

\section{II.SYSTEM MODEL}

We consider a scenario where femtocells are deployed over the existing macrocell network and share the same frequency spectrum with macrocell. Here we focus only on downlink scenario. The downlink communications in a femtocell network consisting of one macrocell and $\mathrm{N}_{\mathrm{F}}=4$ number of femtocells. As shown in Fig.1, a macrocell is at the centre of the network and 4 femtocells are located at $(2,2),(-2,2),(-2,-$ $2),(2,-2)$ within the macrocell coverage. In a macrocell, total $\mathrm{N}_{\text {MUE }}$ user equipments (UEs) are randomly distributed within its coverage area.

Let MBS be the macrocell base station (BS) and $\mathrm{MUE}^{(\mathrm{u})}$ be the $\mathrm{u}$-th user equipment (UE) in the macrocell. Similarly, total $\mathrm{N}_{\mathrm{FUE}}$ number of UEs are randomly distributed in the $\mathrm{j}$-th femtocell, $j \in=\left\{1,2, \ldots \ldots, \mathrm{N}_{\mathrm{F}}\right\}$ and let $\mathrm{FAP}_{\mathrm{j}}$ and $\mathrm{FUE}_{\mathrm{j}}^{(\mathrm{i})}$ be the j-th FAP and the i-th UE in the j-th femtocell, respectively. The RBs are distributed equally among the MUEs of a macrocell and the same resources are reused within every femto-cell. In order to do fair comparision, the evaluation of transmit power per resource block (RB) is done a priori and is kept constant for all systems [7]. The number of UEs and their position in every cell are considered to vary at each time slot to properly consider time varying cell loading. The slot is a time unit during which the loading status remains static and can be specified according to a considered system. For optimum dynamic cell loading, femto cells opportunistically co-use RBs assigned to other cells if the needed average user throughput is not attained with the primarily assigned resource blocks. We assume that there are total $\mathrm{N}_{\mathrm{RB}}$ number of $\mathrm{RBs}$ in the entire network. Here, the macrocell has a priority of determining the number of RBs to serve its own UEs, $\mathrm{N}_{\mathrm{MRB}}$. The remaining $\left(\mathrm{N}_{\mathrm{RB}}-\mathrm{N}_{\mathrm{MRB}}\right) \mathrm{RBs}$ are orthogonally assigned to femto cells so that $\left[\left(\mathrm{N}_{\mathrm{RB}}-\mathrm{N}_{\mathrm{MRB}}\right) / \mathrm{N}_{\mathrm{F}}\right] \mathrm{RBs}$ are assigned to each femtocell.

A) Propagation Path Loss Model(Empirical Model)-

Nearly all radio network systems operate in urban areas where there is no such line of sight (LOS) path between the transmitter and receiver due to the presence of high rise buildings which cause intense diffraction loss [9].

The free space propagation model is modified with some essential corrections and referred to as the 'Plane Earth' propagation [10]. As per our environment Okumura-Hata propagation path loss model for urban area is absolutely perfect [5].

- Macrocell Path Loss Model-

Empirical model is nothing but an excessive computational effort [5]. In Okumura-Hata propagation model the important parameter for us is to know how much overall area is covered. Okumura takes urban areas as a reference and applies correction factors:

$$
L_{d B}=A+B \log _{10}(d)-\mathrm{E}
$$

Where, $A=69.55+26.16 \log _{10} f_{c}-13.82 \log _{10} h_{b}$

$$
\begin{gathered}
B=44.9-6.55 \log _{10} h_{b} \\
E=3.2\left(\log _{10}\left(11.7554 h_{m}\right)\right)^{2} \\
-4.97 \text { for large cities, } f_{c} \geq 300 \mathrm{Mhz} \\
\text { TABLE-I }
\end{gathered}
$$

Some Values Related to Okumura-Hata model [6]

\begin{tabular}{|c|c|c|}
\hline Parameters & Symbol & Range \\
\hline Carrier Frequency & $\mathrm{f}_{\mathrm{c}}$ & $150 \leq \mathrm{f}_{\mathrm{c}} \leq 2000(\mathrm{MHz})$ \\
\hline $\begin{array}{c}\text { BS Antenna } \\
\text { Height }\end{array}$ & $\mathrm{h}_{\mathrm{b}}$ & $30 \leq \mathrm{h}_{\mathrm{b}} \leq 200(\mathrm{~m})$ \\
\hline $\begin{array}{c}\text { MS Antenna } \\
\text { Height }\end{array}$ & $\mathrm{h}_{\mathrm{m}}$ & $1 \leq \mathrm{h}_{\mathrm{m}} \leq 10(\mathrm{~m})$ \\
\hline $\begin{array}{c}\text { Distance Between } \\
\text { BS and MS }\end{array}$ & $\mathrm{d}$ & $1 \leq \mathrm{d} \leq 20(\mathrm{~m})$ \\
\hline
\end{tabular}

\section{- Femtocell Path Loss Model-}

Two different path loss exponents and small breakpoint distance of 100 meters are used to characterize the femtocell path loss propagation model [5]. 
Path Loss:

$$
\begin{aligned}
& L=10 * \propto_{p 1} \log _{10} r+L_{1} \quad \text { for } r<r_{b} \\
& =10 * \propto_{p 2} \log _{10}\left(r / r_{b}\right)+10 * \propto_{p 1} \log _{10} r_{b}+L_{1} \text { for } r>r_{b}
\end{aligned}
$$

where $\mathrm{L}_{1}=$ Reference Path Loss at $\mathrm{r}=1 \mathrm{~m}$.

$\mathrm{r}_{\mathrm{b}}=$ Breakpoint distance

$\propto_{\mathrm{p} 1}=$ Pathloss exponent for $\mathrm{r} \leq \mathrm{r}_{\mathrm{b}}$

$\propto_{\mathrm{p} 2}=$ Pathloss exponent for $\mathrm{r}>\mathrm{r}_{\mathrm{b}}$

To avoid sharp transition between the two regions:

$L=L_{1}+10 * \propto_{p 1} \log _{10} r+10 *\left(\propto_{p 2}-\propto_{p 1}\right) \log _{10}\left(1+r / r_{b}\right)$

\section{B) Radio Channel Model-}

Only MBS can cause interference for FBSU receiver or FBS transmitter. The position of MBS, MBSU, FBS, and FBSU are shown in Fig.1. The utilization of spectrum resources can be improved by cognitive radio (CR) technology. CR facilitates $\mathrm{SU}$ to access the spectrum bands licensed to PU as long as PUs QoS is not affected. Here, MBSU is treated as PU and FBSU is treated as SU. PU is power controlled by the BS.The link gain between MBS and $\mathrm{u}$-th UE can be expressed as:

$$
G_{p 2 p, u}\left(r_{u}, \theta_{u}\right)=d_{u}{ }^{-\alpha_{p}} 10^{\xi_{s} / 10}
$$

where $d_{u}\left(r_{u}, \theta u\right)$ is denoted as the distance of a MBSU /PU at a location $\left(r_{u}, \theta u\right)$ with respect to the MBS. We have neglected the co-tier interference as FBSs are deployed in such a manner that it eliminates all the difficulties of overlapping of FBSs. The link gain between $\mathrm{j}$-th FBS and i-th FBSU can be expressed as:

$$
G_{s 2 s, j i}=d_{s 2 s, j i}^{-\propto_{p}} 10^{\xi_{s}} / 10
$$

where $d_{s 2 s, j i}$ is the distance between $\mathrm{j}$-th FBS and i-th FBSU. $\xi_{\mathrm{s}}$ (in $\mathrm{dB}$ ) is a Gaussian random variable with zero mean and variance, ${ }^{2}$, due to shadowing in the channel.

A FBSU is assumed to be within a circle of radius, $r_{f}$ around a FBS. If the position of a FBS is $\left(\mathrm{r}_{\mathrm{j}}, \theta \mathrm{j}\right)$ and the position of a FBSU is $\left(r_{i}, \theta i\right)$. Then,

$$
d_{s 2 s, j i}=\sqrt{\left(r_{j} \cos \theta_{j}-r_{i} \cos \theta_{i}\right)^{2}+\left(r_{j} \sin \theta_{j}-r_{i} \sin \theta_{i}\right)^{2}}
$$

If the position of a MBS is $\left(r_{k}, \theta k\right)$ then the distance of it from FBSU may be expressed as-

$$
d_{p 2 s, k i}=\sqrt{\left(r_{k} \cos \theta_{k}-r_{i} \cos \theta_{i}\right)^{2}+\left(r_{k} \sin \theta_{k}-r_{i} \sin \theta_{i}\right)^{2}}
$$

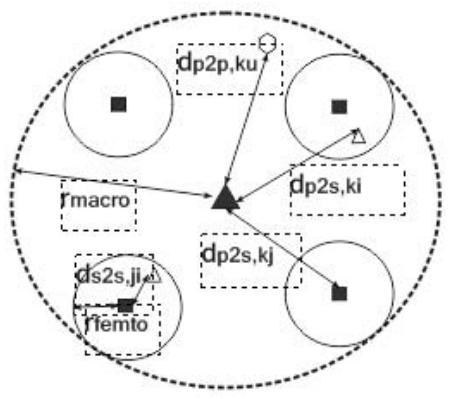

Fig.2 Computation of distance/path from the position of a user (PU/MBSU or SU/FBSU) to MBS and FBS.
The link gain from k-th MBS to i-th FBSU may be expressed as:

$$
G_{p 2 s, k i}=d_{p 2 s, k i}^{-\propto_{p}} 10^{\xi_{s} / 10}
$$

where $d_{p 2 s, ~ k i}$ is the distance between k-th MBS to i-th FBSU. All the channel gains are assumed to be independent and the channels have a coherence time greater than or equal to a time slot. In this work, we have not considered fading and shadowing effect. We have considered pathloss only.

We will use the notation $\mathrm{x}$ to denote the serving network entity for a generic user. That is, $x=\mathrm{a}$ if the user is associated to a FAP and $x=\mathrm{b}$ if the user is associated to a MBS. Without any loss of generic laws, the analysis is conducted on a typical user located at the origin [11]. Therefore, the signal to interference plus noise ratio (SINR), $\gamma_{x}$ at the typical user located at the origin (which also holds for any generic user) served by an MBS or FAP (MBS/FAP) is given by

$\gamma_{x}=\frac{P_{x} D_{x}^{-\alpha_{p}}}{\sum_{b_{i} \in \widetilde{\psi}_{b_{x}}} P_{b} b_{i}^{-\alpha_{p}}+\sum_{a_{i} \in \widetilde{\psi}_{a_{x}}} P_{a} a_{i}^{-\alpha_{p}}+N}$

where $\mathrm{D}_{x}$ is the distance from the user to the serving network entity (i.e. an MBS or a FAP), $\tilde{\psi}_{b_{x}}$ denotes the set of interfering MBSs, $\tilde{\psi}_{a_{x}}$ denotes the set of interfering FAPs and $\sigma^{2} i$ s the noise power[12].

C) Outage Probability for Downlink Transmission of macro user-

A FAP (or equivalently its associated user) experiences two kinds of outages. The first is due to the channel unavailability because of the opportunistic channel access. While the second is the SINR outage. According to practical scenario, there is existence of SISO outage users and SIMO outage users. A user is said to be in outage if SINR of the user is less than a SINR threshold.

The outage probability of a SISO / SIMO may be expressed as:

$$
\begin{aligned}
& P_{\text {out }, \text { SISO }}=\operatorname{Prob}\left\{\gamma_{x} \leq \operatorname{SIN} R_{\text {thd }(\text { SISO })}\right\} \\
& P_{\text {out }, \text { SIMO }}=\operatorname{Prob}\left\{\gamma_{x} \leq \operatorname{SIN} R_{\text {thd }(\text { SIMO })}\right\}
\end{aligned}
$$

\section{D) Throughput of Macrocell Network-}

We assume that exact synchronisation is there in time and frequency domain, therefore, interference among the surrounding/nearest RBs is neglected. The reachable throughput, $\mathrm{T}_{\mathrm{p}}$ of an UE can be calculated from Shannon's theorem. Considering a bandwidth $\mathrm{W}$ is allocated to macrocell networks. we have

$$
T_{p}=W \log _{2}\left(1+\gamma_{x}\right)
$$

where $\gamma_{x}$-Signal to Interference plus Noise Ratio

\section{III.SIMULATION MODEL}


The simulation is developed in MATLAB. In our simulation, parameters mentioned in Table 1 and Table 2 are used. For simplicity of analysis we consider the pathloss only.

\section{A. Generation of Users' Locations and Interference Powers}

The generation of the users' locations and interference powers is carried out considering the following steps.

1. A fixed number of users $\left(\mathrm{N}_{\mathrm{UE}}=\mathrm{N}_{\mathrm{MUE}, \mathrm{u}}+\mathrm{N}_{\mathrm{FUE}, \mathrm{ji}}\right)$ is generated and they are randomly distributed within the coverage area . This includes all MBSUs /PUs $\left(\mathrm{N}_{\mathrm{MUE}, \mathrm{u}}\right)$ and FBSUs $/ \mathrm{SU}_{\mathrm{s}}\left(\mathrm{N}_{\mathrm{FUE}, \mathrm{ji}}\right)$. Here, $j \in=\left\{1,2, \ldots \ldots ., \mathrm{N}_{\mathrm{F}}\right\} ; \quad \mathrm{m}=60$, $u \forall\left\{1,2,3 \ldots \ldots m R_{b}\right\}, \quad \mathrm{R}_{\mathrm{b}} \quad$ No. of resource block(RB); $i \forall\{1,2,3, \ldots . n\}, n \in$ any large integer value .

2. The locations (in the $(r, \theta)$ coordinate system) of all SUs and PUs $\left(\mathrm{N}_{\mathrm{UE}}\right)$ are generated within the coverage of macrocell. $\mathrm{N}_{\mathrm{FUE}, \mathrm{ji}}$ number of $\mathrm{SU}$ receivers are generated around $\mathrm{j}$ number of corresponding SU transmitters (FBS/FAP). The SU receivers are present around a SU transmitter, within a circle of radius, $r_{\text {f emto }}$.

3. For each of the $\mathrm{N}_{\mathrm{UE}}$ users, the link gains corresponding to BS and UE are generated, following the formulas derived in Section II.

4. The received signal strength (RSS) is evaluated from $\mathrm{PU} / \mathrm{MBSU}$ or SU/FBSU at the reference MBS or FBS. Similarly, interference power at a PU receiver or SU receiver is evaluated, considering all the possibilities.

5. Next, the SINR for a PU/Macro user and/or a SU/Femto user are computed.

B) Probability of Outage for MBSUs and FBSUs-

The following steps are considered.

1. The SINR is generated for a desired user as shown in the previous section and compared with a threshold value given by $S I N R_{\text {thd (SISO) }}$ and $S I N R_{\text {thd (SIMO) }}$.

2. If the $\gamma_{x}$ falls below $S I N R_{\text {thd(SISO) }}$, an outage counter (outage count(SISO) $_{\text {) is incremented. }}$

Similarly, If the $\gamma_{x}$ falls below $S I N R_{t h d(\operatorname{SIMO})}$, an outage

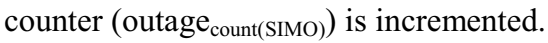

3. Steps (2) and (3) are repeated a large $\left(\mathrm{N}_{t}>>1\right)$ number of times to yield an accurate estimate of the probability of outage as $\quad P_{\text {out,SISO }}=\left(\right.$ outage $\left.\mathrm{e}_{\text {count }(\text { SISO })} / \mathrm{N}_{\mathrm{t}}\right)$ and $P_{\text {out }, \text { SIMo }}=\left(\right.$ outage $\left.\mathrm{e}_{\text {count }(\text { SIMO })} / \mathrm{N}_{\mathrm{t}}\right)$. Please note that outage probabilities for a MBSU and a FBSU are evaluated in the same way.

\section{RESUlTS AND DisCUSSIONS}

The main parameters of the simulation framework are set as shown in Table 1. \& Table 2.

In Fig. $3 \& 4$, the outage probability for SISO users and SIMO users are shown as a function of the number of UEs (either PU or SU). It is observed that the probability of outage for either SISO or SIMO users increases for increasing values of the number of UEs (either PUs or SUs). This is due to increase in multiple access interference. Obviously, the probability of outage reduces when less number of MBSU is considered. It is also found that probability of outage for SISO and SIMO users have gradually elevated with increase in MBS coverage radius, while keeping BS antenna transmit power same. Noticeable point in Fig. 4 is that minimum outage probability of SIMO user at MBS coverage radius $=3 \mathrm{Km}$ and there is not much change in slope with the increasing number of UE.

In Fig. $5 \& 6$, the probability of outage for SISO and SIMO users are shown as a function of macrocell radius. Probability of outage for SISO or SIMO user increases with increase in macrocell radius. Note that, all FBSs are assumed to have same antenna transmit power. We have also shown the effect on outage probability by considering different FBS power (antenna transmit power), while keeping MBS power fixed.

The result shows that, quite a few instances when MBSU suffering from FBS interference due to implantation of FBS, but at the same time FBS provides network coverage which helps to reduce overall outage of the networks.

Normally, any macrocell user (MBSUs) who experiences/faces interference would be switched to a different channel and would avoid outage from the femtocells.

The probability of outage for a SISO or SIMO user is higher when macrocell radius is higher. However, the probability of outage of a SISO or a SIMO user does not change much with distance beyond $16 \mathrm{~km}$.

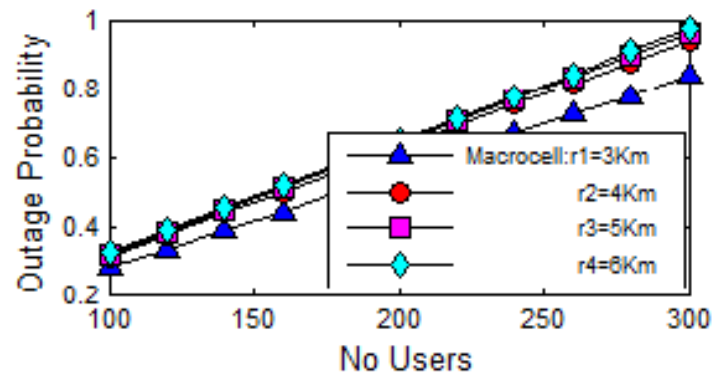

Fig.3 Probability of outage for SISO users vs. the number of UEs

In Fig. $7 \& 8$, throughput of macrocell network for SISO and SIMO users are shown as a function of the number FBSUs. Throughput of macrocell network decreases with increase in number of FBSUs. Three different values of antenna transmit power for UEs are considered in this analysis.

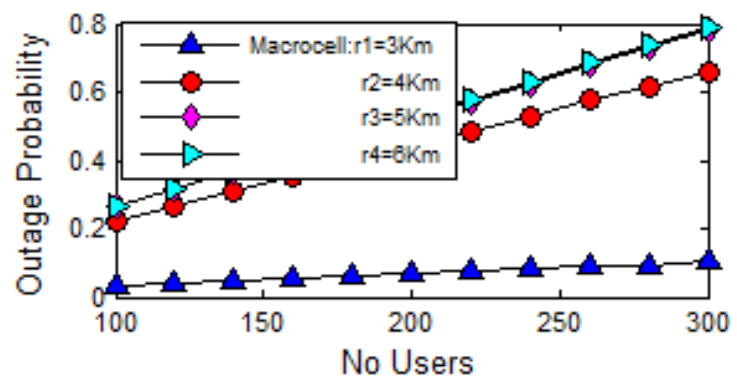

Fig.4 Probability of outage for SIMO users vs. number of UEs

In Fig.7, the measure of throughput performance is taken by assuming different MBS transmit power, while keeping FBS power fixed. Fig. 8 highlights the throughput performance by considering different FBS transmit power, while keeping MBS power fixed. 


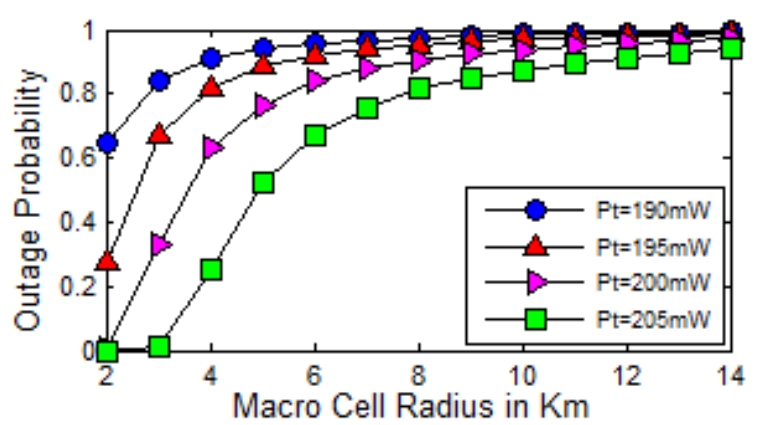

Fig. 5 Probability of outage for SISO users vs. macrocell radius

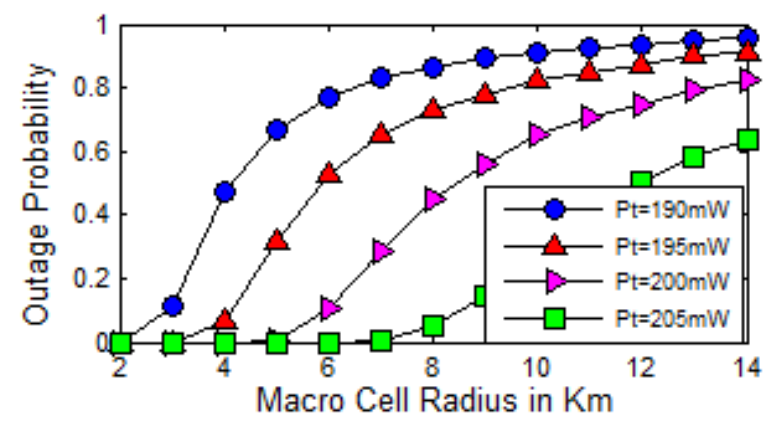

Fig.6 Probability of outage for SIMO users vs. macrocell radius

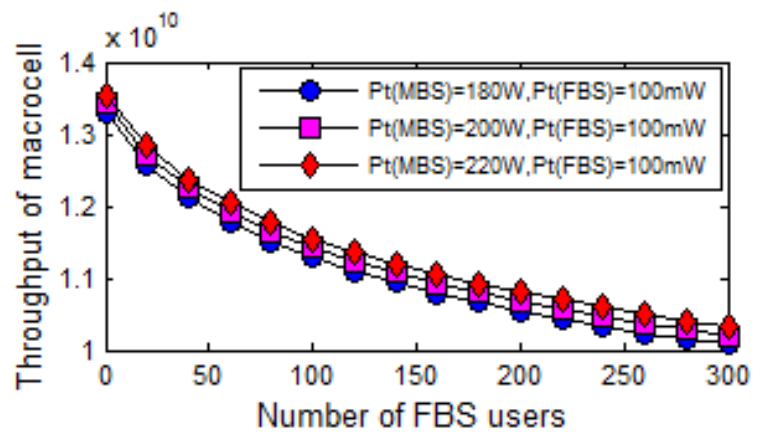

Fig.7 Throughput of macrocell network vs. number of FBSUs for different MBS transmit power

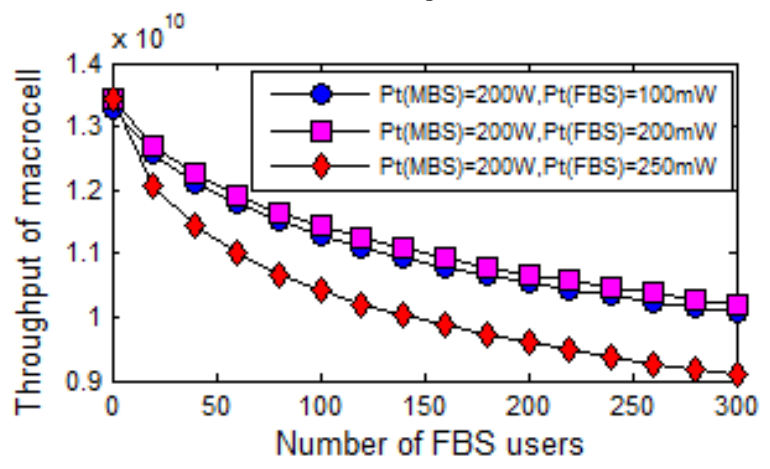

Fig. 8 Throughput of macrocell network vs. the number of FBSUs for different MBS transmit power

\section{V.CONCLUSION}

In this paper, we develop a simulation testbed for performance analysis of cognitive femtocell network. We propose a simplified network model. The overall outage performance of SISO and SIMO user improves as their (FAP) intensity increases and/or their transmission power increases. This is because, increasing the intensity and transmission powers of the FAPs, while keeping the parameters of the FBSs constant, enhances the useful signal with respect to the intertier interference. More precisely, the outage probabilities of SISO and SIMO users have been compared for various values of network parameters. For the given scheme, our results suggest that the throughput depends strongly on the intensity and transmission powers of FAPs; whereas antenna transmission power of MBS has a limited impact. The cognitive femtocell is an important feature which embeds into the network to boost up the overall system performance. Future work includes evaluation of performance in the presence of fading and shadowing.

\section{REFERENCES}

[1] http://www.smallcellforum.com

[2] J.G. Andrews, and A.Gatherer,"Femto networks: a Survey", IEEE Commun. Mag., vol.46, no.9, pp.59-67, Sept.2008.

[3] Simon R. Saunders, Stuart Carlaw, Andrea Giustina, Ravi Raj Bhat, Srinivasa RaoRasa Siegberg," Femtocells: Opportunities and Challenges for Business and Technology," (c) 2009 John Wiley \& Sons Ltd. ISBN: 978-0-47074816-9.

[4]3GPP TS36.300 V9.0.0,"Evolved universal terrestrial radio access (EUTRA) and evolved universal terrestrial radio access network (EUTRA),"2009.

[5]Sylvain Ranvier, "Path loss models: S-72.333 Physical layer methods in wireless communication systems", Helsinki University of Technology, Smarad Centre of Excellence, 23 November 2004.

[6] D. Lopez-Perez, A. Valcarce, G. De La Roche, and J. Zhang, "OFDMA femtocells: a roadmap on interference avoidance," IEEE Communications Magazine, vol. 47, no. 9, pp. 41 - 48, Sep. 2009.

[7] Zubin and Harald Haas,"Throughput Enhancement Through Femtocell Deployment," European Transaction on Telecommunication, vol. 21 no. 4, 2010.

[8] S. Haykin, "Cognitive Radio: Brain-Empowered Wireless Communications," IEEE JSAC, vol. 23, no. 2, Feb. 2005, pp. 201-20.

[9] Dennis J. Dupray, Charles L. Karr," Geographic location using multiple location estimators", Publication number US7298327B2, 20 Nov 2007.

[10] Ian Wassell and Yan Wu,"Radio Propagation Modelling," University of Cambridge Computer Laboratory.

[11] J Andrews, F.Baccelli, and R.Ganti,"A tactable approach to coverage and rate in cellular network", IEEE Transaction on Communications, vol.59, no.11, pp.-3122-3134, November 2011.

[12] M. Haenggi and R. Ganti,"Interference in large wireless networks," in Foundations and Trends in Networking. now Publishers, 2008, vol.3, no.2, pp. $127-248$

TABLE 2

Network Based Parameters Used in Simulations

\begin{tabular}{|l|l|l|}
\hline \multicolumn{2}{|l|}{ Parameters } & Values/Range \\
\hline \multicolumn{2}{|l|}{ Antenna type } & Omni-directional \\
\hline \multirow{2}{*}{ Cell Radius } & Macrocell & $6 \mathrm{Km}, 8 \mathrm{Km}, 10 \mathrm{Km}, 12 \mathrm{Km}$ \\
\cline { 2 - 3 } & Femtocell & $1000 \mathrm{~m}$ \\
\hline \multirow{2}{*}{$\begin{array}{l}\text { Antenna } \\
\text { height }\end{array}$} & $\mathrm{h}_{\mathrm{t}}$ & $20 \mathrm{~m}, 50 \mathrm{~m}, 80 \mathrm{~m}$ \\
\cline { 2 - 3 } & $\mathrm{h}_{\mathrm{r}}$ & $0.5 \mathrm{~m}, 1 \mathrm{~m}, 1.8 \mathrm{~m}$ \\
\hline \multicolumn{2}{|c|}{ Transmit antenna gain } & 13 \\
\hline Path loss exponent, $\alpha_{\mathrm{p}}$ & 4 \\
\hline Shadowing standard deviation, & 4 \\
\hline Noise spectral density( $\left.\eta_{0}\right)$ & $-204 \mathrm{~dB}$ \\
\hline Carrier frequency, $\mathrm{f}_{\mathrm{c}}$ & $1900 \mathrm{MHz}$ \\
\hline No of Resource Block(RB) & 100 \\
\hline \multicolumn{2}{|l|}{ System Noise Figure } & $5 \mathrm{~dB}$ \\
\hline $\begin{array}{l}\text { SINR } \\
\text { threshold }\end{array}$ & SINR & $15 \mathrm{~dB}$ \\
\cline { 2 - 3 } thd(SISO) & SINR $_{\text {thd(SIMO) }}$ & $12.5 \mathrm{~dB}$ \\
\hline
\end{tabular}

